\title{
The challenge of obesity treatment: avoiding weight regain
}

\author{
Robert Ross PhD
}

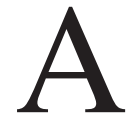
lthough lifestyle-based programs for treatment of obesity are generally associated with weight loss of about $10 \%,{ }^{1}$ avoiding weight regain is a major challenge. ${ }^{2}$ Most patients regain about $30 \%$ of the weight lost in the year following treatment and typically return to their baseline weight in 3-5 years. ${ }^{2}$ In this issue, Dale and colleagues ${ }^{3}$ report the results of a 2-year randomized controlled trial that tested 2 questions: does the intensity of support provided by health professionals affect the maintenance of weight loss, and are differences in the consumption of macronutrients related to the maintenance of previous weight loss.

Dale and colleagues recruited 200 overweight and obese women who had lost $5 \%$ or more of their initial body weight in the 6 months before enrollment. Participants were randomly assigned to receive intensive support or nurse support and were prescribed a diet high in carbohydrate or monounsaturated fat. Women in both programs maintained a mean weight loss of $8 \%$ over the 2-year follow-up period. The authors concluded that, because women in both groups maintained their weight, the more expensive intensive-support program was not necessary.

These findings contradict those of previous randomized studies in which participants regained weight despite relatively intensive support. In a 30-month study that included 1032 overweight or obese men and women who had lost at least $4 \mathrm{~kg}$ during a 6-month weight-loss program, Svetkey and colleagues ${ }^{4}$ randomly assigned participants to receive monthly personal contact, unlimited access to an interactive Internet-based intervention or self-directed control. Although most of the participants maintained a weight below their initial weight, all groups regained a mean of $4.0-5.5 \mathrm{~kg}$. The Study to Prevent Regain (STOP), ${ }^{5}$ which included 314 overweight or obese men and women who had lost a mean of $19 \mathrm{~kg}$ in the previous 2 years, found that participants in all groups (monthly face-to-face contact, Internet-based intervention or quarterly newsletters) regained a mean of $2.5-4.9 \mathrm{~kg}$. The findings from these studies reinforce the challenges in the maintenance of weight loss and make the findings of Dale and colleagues remarkable in comparison.

A post-hoc analysis of the STOP trial reported that increased frequency of self-weighing and increased physical activity are predictors of weight maintenance. ${ }^{6}$ In the study by Dale and colleagues, participants in the nurse-support group met with the nurse coordinator for 5 to 10 minutes every 2 weeks and had telephone contact with the nurse on alternate weeks. The frequency of contact and the requirement to self-report or objectively measure body weight was much greater in this trial than

\section{Key points}

- Avoiding weight regain is a challenge for many patients.

- Continued contact with a health care provider after weight loss is beneficial.

- Behavioural factors are associated with sustained weight loss.

- Exercise specialists and dieticians can provide support for sustained weight loss.

- Simple pragmatic programs may suffice.

in previous trials. ${ }^{4,5}$ This underscores the notion that self-weighing combined with face-to-face contact and continued dialogue is important for long-term maintenance of weight loss.

Surprisingly, Dale and colleagues observed that, regardless of the type of support, the participants' physical activity levels did not change during the study and, thus, did not account for the maintenance of weight. This finding is in contrast with the dogma that increasing physical activity is a strong, independent predictor of the success of maintenance of weight loss. Their findings instead support the conclusion that counselling about physical activity is not an essential component of programs for the maintenance of weight. This is a disappointing result, given the observation that increasing physical activity and cardiorespiratory fitness are associated with substantial reductions in obesity-related morbidity and mortality, independent of body weight. ${ }^{7}$ However, results from a recent study suggest that a pragmatic nurse-coordinated program designed to increase the levels of physical activity of overweight and obese participants successfully achieved marked and sustained increases in physical activity over 2 years. ${ }^{8}$ Perhaps the focus by Dale and colleagues on the maintenance of weight as the determinant of success deflected the participants' attention and enthusiasm away from physical activity. Regardless, it is encouraging that both studies ${ }^{3,8}$ found that the behaviours most often associated with sustained weight loss (self-weighing, reducing calories and increasing physical activity) may be achieved in a cost-effective way.

The finding by Dale and colleagues that the consumption of macronutrients did not affect the maintenance of weight loss is consistent with the finding of Sacks and colleagues, ${ }^{9}$ who reported that calorie-reduced diets resulted in similar amounts of weight loss and maintenance regardless of which macronutrients were emphasized. Thus, behavioural factors

Robert Ross is with the School of Kinesiology and Health Studies, Medicine, Division of Endocrinology and Metabolism, Queen's University, Kingston, Ont. 
(caloric restriction), rather than metabolism of macronutrients, are associated with the maintenance of weight loss.

Although intensive-support programs may be more costly than nurse-supported programs, the true cost and, more importantly, the cost-effectiveness of these programs are unknown. The feasibility of implementing nurse-coordinated programs within different settings is unclear. Challenges in Canada include a paucity of nurses, who are already faced with heavy workloads. The nurse in the study by Dale and colleagues had little training in nutrition and exercise but was supportive and enthusiastic. Thus, the content, approach and frequency of care provided in support programs may be more important than who provides it. The findings of Dale and colleagues support the inclusion of exercise specialists and dieticians in the care of overweight and obese adults, because these health professionals have the relevant training, are no more expensive than nurses, and would be enthusiastic supporters of their nursing colleagues. Dale and colleagues deserve much credit for showing that clinically meaningful weight loss can be maintained for an extended period in a pragmatic manner. Although the challenges inherent to the treatment of obesity are real, so too are the opportunities.

\section{REFERENCES}

1. Lau DC, Douketis JD, Morrison KM, et al.; for the Obesity Canada Clinical Practice Guidelines Expert Panel. 2006 Canadian clinical practice guidelines on the management and prevention of obesity in adults and children [executive summary]. CMAJ 2007; 176:S1-13.

2. Weiss EC, Galuska DA, Kettel Khan L, et al. Weight regain in U.S. adults who experienced substantial weight loss, 1992-2002. Am J Prev Med 2007;33:34-40.

3. Dale KS, McAuley KA, Taylor RW, et al. Determining optimal approaches for weight maintenance: a randomized controlled trial. CMAJ 2009;180:E39-E46.

4. Svetkey LP, Stevens VJ, Brantly PJ, et al.; Weight Loss Maintenance Collaborative Research Group. Comparison of strategies for sustaining weight loss: the weight loss maintenance randomized controlled trial. JAMA 2008;299:1139-48.

5. Wing RR, Tate DF, Gorin AA, et al. A self-regulation program for maintenance of weight loss. $N$ Engl J Med 2006;335:1563-71.

6. Wing RR, Papandonatos G, Fava JL, et al. Maintaining large weight losses: the role of behavioral and psychological factors. J Consult Clin Psychol 2008;76: 1015-21.

7. Ross R, Janiszewski PM. Is weight loss the optimal target for obesity-related cardiovascular disease risk reduction? Can J Cardiol 2008;24 Suppl D:25D-31D.

8. Lawton BA, Rose SB, Raina Elley C, et al. Exercise on prescription for women aged 40-74 recruited through primary care: two year randomised controlled trial. Br J Sports Med 2009;43:120-3.

9. Sacks FM, Bray GA, Carey VJ, et al. Comparison of weight-loss diets with different compositions of fat, protein, and carbohydrates. N Engl J Med 2009;360:859-73.

Correspondence to: Dr. Robert Ross, School of Kinesiology and Health Studies, Medicine, Division of Endocrinology and Metabolism, Queen's University, 69 Union St., Kingston ON

K7L 3N6; rossr@post.queensu.ca

\section{What kind of randomized trials do we need?}

\section{Merrick Zwarenstein MD MSc, Shaun Treweek BSc PhD}

Published at www.cmaj.ca on Apr. 16, 2009. This article was published simultaneously in the May 2009 issue of the Journal of Clinical Epidemiology (www.jclinepi.com).

$\infty$ See related article by Thorpe and colleagues, page E47, and commentary by Maclure and colleagues, page 1001

I n 1967, Daniel Schwartz and Joseph Lellouch, 2 French statisticians, and their British colleague and translator Michael Healy wrote "[M]ost therapeutic trials are inadequately formulated, and this from the earliest stages of their conception."

The seminal paper ${ }^{1}$ from which this dramatic assertion is drawn is reprinted in the May 2009 issue of the Journal of Clinical Epidemiology as part of a joint focus with CMAJ on making randomized controlled trials (RCTs) more useful.

Schwartz and Lellouch argued that there are 2 kinds of randomized trials embodying radically different attitudes to evaluation of treatment, which they named "pragmatic" and "explanatory." They go on to say that these 2 attitudes require different approaches to the design of a randomized trial. The pragmatic attitude seeks to directly inform realworld decisions among alternative treatments. Schwartz and Lellouch show that this purpose is satisfied in trials that se- lect typical participants, settings and comparator care to widen real-world applicability. In contrast, the explanatory attitude seeks to understand a biological process by testing the hypothesis that the specified biological response is explained by exposure to a particular treatment. Tight restrictions on participants, treatment, control and setting maximize the contrast with the control group and increase the ability to test this kind of hypothesis.

Their assertion of inadequate formulation relates to the mismatch between the use we make of most trials (which is to inform decisions on therapy) and the design of these trials (which generally takes the opposite form, best suited to test-

Merrick Zwarenstein is with the Centre for Health Services Sciences, Sunnybrook Research Institute, Toronto, Ont. Shaun Treweek is with the Division of Clinical and Population Science and Education, University of Dundee, Dundee, UK. 